This is the author's final, peer-reviewed manuscript as accepted for publication. The publisher-formatted version may be available through the publisher's web site or your institution's library.

\title{
Accelerate Sampling in Atomistic Energy Landscapes Using Topology-Based Coarse-Grained Models
}

Weihong Zhang and Jianhan Chen

\section{How to cite this manuscript}

If you make reference to this version of the manuscript, use the following information:

Zhang, W., \& Chen, J. (2014). Accelerate sampling in atomistic energy landscapes using topology-based coarse-grained models.

\section{Published Version Information}

Citation: Zhang, W., \& Chen, J. (2014). Accelerate sampling in atomistic energy landscapes using topology-based coarse-grained models. Journal of Chemical Theory and Computation, 10(3), 918-923.

Digital Object Identifier (DOI): 10.1021/ct500031v

Publisher's Link: http://pubs.acs.org/doi/abs/10.1021/ct500031v

This item was retrieved from the K-State Research Exchange (K-REx), the institutional repository of Kansas State University. K-REx is available at http://krex.ksu.edu 


\title{
Accelerate sampling in atomistic energy landscapes using topology- based coarse-grained models
}

\author{
Weihong Zhang and Jianhan Chen* \\ Department of Biochemistry and Molecular Biophysics \\ Kansas State University \\ Manhattan, KS 66506, USA
}

Submitted to Journal of Chemical Theory and Computation as a Letter

Revised Version

Corresponding Author: Phone: (785) 532-2518; Fax: (785) 532-7278; Email: jianhanc@ksu.edu 


\begin{abstract}
We describe a multi-scale enhanced sampling (MSES) method where efficient topology-based coarse-grained models are coupled with all-atom ones to enhance the sampling of atomistic protein energy landscape. The bias from the coupling is removed by Hamiltonian replica exchange, thus allowing one to benefit simultaneously from faster transitions of coarse-grained modeling and accuracy of atomistic force fields. The method is demonstrated by calculating the conformational equilibria of several small but nontrivial $\beta$-hairpins with varied stabilities.
\end{abstract}

Keywords: multi-scale, enhanced sampling, implicit solvent, protein folding, replica exchange, conformational ensemble, hairpin 
Generating statistically representative conformational ensembles remains a major challenge in atomistic simulation of biomolecules ${ }^{1}$. This is not only due to the large and complex conformational space, but also because of significant energy barriers that frequently separate different subspaces. Temperature replica exchange (T-RE) ${ }^{2-4}$ is now widely accepted as a relatively straightforward yet powerful technique for enhanced sampling. Multiple replicas of the system are simulated independently at different temperatures, and periodically attempt to exchange simulation temperatures according to a Metropolis criterion that preserves the detailed balance. The resulting random walk in the temperature space helps each replica to escape local energy minima and thus facilitate conformational sampling. Extensive theoretical and simulation studies have confirmed that T-RE enhances sampling compared to constant temperature simulations as long as the activation enthalpies of conformational transitions are positive ${ }^{5-9}$. Nonetheless, the efficiency of T-RE can be severely limited by the presence of sharp cooperative conformational transitions such as protein folding ${ }^{10,11}$. Importantly, this limitation cannot be overcome by various T-RE variants designed to accelerate either exchanges or diffusion in temperature space ${ }^{12}$. In practice, virtually all T-RE protein simulations involve exchange attempt frequencies $\left(\sim \mathrm{ps}^{-1}\right)$ that are several orders of magnitude faster than the slowest protein motions (folding; $\mu \mathrm{s}^{-1}$ or slower ${ }^{13}$ ). As such, the efficiency of T-RE sampling of large-scale conformational transitions is rarely limited by diffusion in temperature space, but mainly by the inherent rates of spontaneous processes.

Fundamentally, the limited efficiency of T-RE in sampling cooperative transitions such as protein folding can be attributed to large entropic components in the free energy barriers ${ }^{14,15}$. The folding rate only depends weakly on temperature and often displays anti-Arrhenius behaviors ${ }^{5,16}$, such that tempering is ineffective in driving transitions. Instead, coarse-grained (CG) models ${ }^{17}$ are often utilized to significantly reduce the conformational space and allow faster reversible transitions; but this is achieved at the expense of reduced detail and accuracy. An ideal approach could involve CG modeling to overcome major entropic barriers and at the same time seamlessly propagates the transitions to atomistic simulations for detailed sampling of different conformational subspaces. A key requirement is that one must be able to recover canonical ensembles at the atomistic level. The promise of such multi-scale enhanced sampling (MSES) has been well recognized, and several clever ideas have been proposed towards this goal ${ }^{18-23}$. The resolution exchange approach is particularly interesting ${ }^{19}$. It involves independent simulations of the system at two or more resolutions and attempts to directly swap shared coordinates of low- and high-resolution models 
according to Metropolis criteria. Resolution exchange in principle allows the possibility of injecting high-resolution simulation into novel conformational subspaces sampled at low-resolution. A key limitation is that conformations sampled at different resolutions must be similar to be exchangeable for large biomolecules, as excessive bad contacts would effectively prohibit successful exchange. A smart resolution replica exchange was proposed to improve exchange acceptance, where a ladder of mixed atomistic and $\mathrm{CG}$ potentials are used and $\mathrm{CG}$-sampled configurations are relaxed prior to exchange attempt ${ }^{24}$. However, the detailed balance is broken due to relaxation and only canonical sampling is achieved. More importantly, the aggressive approach of direct coordinate swap in resolution exchange, with or without relaxation, requires configurations sampled at different resolutions to closely track each other, which counteracts the purpose of rapid hopping among drastically different conformational subspaces sampled at the CG level.

The efficacy of an MSES scheme, regardless of how sampling at different resolutions is coupled, depends critically on the ability of CG simulations to generate transitions that are consistent with inherent conformational dynamics of the atomistic model. Otherwise, CG simulations would attempt to engage the atomistic model along unfavorable pathways and become ineffective in accelerating atomistic transitions. For proteins, topology-based CG modeling has been highly successful and demonstrated impressive correspondence between experiment and theory for folding mechanisms ${ }^{25}$. This approach is based on to the minimal frustration theory of protein folding ${ }^{26}$, which argues that native interactions dictate the protein free energy landscape and that stabilization due to non-native contacts ("frustration") should be minimal. Accordingly, the true protein energy landscape can be approximated by effective energy functions that only include native interactions. These effective energy functions, commonly referred to as Gō or Gō-like models, are highly efficient, and yet powerful enough to generate realistic reversible folding pathways. As such, they should be ideally suitable for driving conformational sampling on atomistic protein energy landscapes. Here, we describe an MSES approach that utilizes the efficient sequence-flavored Gōlike model ${ }^{27}$ to accelerate the sampling of atomistic protein conformational equilibria, and demonstrate its efficiency using a series of small but nontrivial $\beta$-hairpins with varied stabilities.

Our approach is inspired by Moritsugu et al.'s multi-scale essential sampling method ${ }^{20}$, where both the $\mathrm{CG}$ and atomistic representations of the protein are simulated simultaneously in a hybrid system. The atomistic and CG copies do not interact, and are only coupled through restraint potentials designed to restrict the divergence between $\mathrm{CG}$ and atomistic configurations. The overall potential function of the hybrid system is 


$$
U_{\mathrm{mix}}\left(\boldsymbol{r}_{\mathrm{AT}}, \boldsymbol{r}_{\mathrm{CG}}, \lambda\right)=U_{\mathrm{AT}}\left(\boldsymbol{r}_{\mathrm{AT}}\right)+U_{\mathrm{CG}}\left(\boldsymbol{r}_{\mathrm{CG}}\right)+\lambda U_{\mathrm{MSES}}\left(\boldsymbol{r}_{\mathrm{AT}}, \boldsymbol{r}_{\mathrm{CG}}\right),
$$

where $U_{\mathrm{AT}}$ and $U_{\mathrm{CG}}$ are the atomistic and CG potential functions, respectively. Only the coupling potential $U_{\text {MSES }}$ depends on both atomistic $\left(\boldsymbol{r}_{\mathrm{AT}}\right)$ and $\mathrm{CG}\left(\mathbf{r}_{\mathrm{CG}}\right)$ coordinates. Given a proper coupling potential, the atomistic and CG copies can be restrained to track one and another when the overall coupling scaling factor $\lambda=1$. Hamiltonian RE can be performed to communicate coupled conformational dynamics to the limit of $\lambda=0$, where the CG and atomistic copies are fully independent and proper canonical ensembles are generated simultaneously at both resolutions. Our MSES approach incorporates both Hamiltonian and temperature RE to further accelerate conformational sampling. Specifically, $N$ replicas of the hybrid system are simulated independently with increasing couple scaling factors and temperatures, $\left\{\lambda_{i}, T_{i}\right\}, i=1,2, \ldots, N . \lambda_{1}=0$ and $\lambda_{\mathrm{N}}=1$. Replicas periodically attempt to exchange simulation conditions according to

$$
P_{m \leftrightarrow n}=\min \left(1, e^{\Delta_{m n}}\right)
$$

where $\Delta_{m n}=\beta_{m}\left[U_{\text {mix }}\left(\boldsymbol{r}_{\mathrm{AT}, m}, \boldsymbol{r}_{\mathrm{CG}, m}, \lambda_{m}\right)-U_{\mathrm{mix}}\left(\boldsymbol{r}_{\mathrm{AT}, n}, \boldsymbol{r}_{\mathrm{CG}, n}, \lambda_{m}\right)\right]+\beta_{n}\left[U_{\mathrm{mix}}\left(\boldsymbol{r}_{\mathrm{AT}, n}, \boldsymbol{r}_{\mathrm{CG}, n}, \lambda_{n}\right)-\right.$ $\left.U_{\text {mix }}\left(\boldsymbol{r}_{\mathrm{AT}, m}, \boldsymbol{r}_{\mathrm{CG}, m}, \lambda_{n}\right)\right]$ and $\beta=1 / k_{\mathrm{B}} T\left(k_{\mathrm{B}}\right.$ is the Boltzmann constant). When the same temperature is used for all replicas, the exchange probability is determined by the coupling term only, allowing excellent scalability to large systems ${ }^{20}$.

Coupling the $\mathrm{CG}$ and atomistic models using restraint potentials is a significant advantage compared to direct coordinate swapping in resolution exchange. It allows one to control the impacts of large divergences between CG and atomistic configurations on the total energy, which dramatically improves exchange efficiency and provides superior scalability to large systems. Motivated by the notion that native contacts dictate protein folding transitions ${ }^{26}$, the $\mathrm{CG}$ and atomistic copies are coupled by penalty functions that depend on the differences in $\mathrm{C} \alpha-\mathrm{C} \alpha$ distances of residues involved in all native contacts:

$$
U_{\mathrm{MSES}}=\sum E_{i}\left(\Delta d_{i}\right)=\sum \frac{1}{2} k_{i}\left(d_{i}^{A T}-d_{i}^{C G}\right)^{2}
$$

where $\Delta d_{i}=d_{i}^{A T}-d_{i}^{C G}$, and $d_{i}^{A T}$ and $d_{i}^{C G}$ are the $\mathrm{C} \alpha-\mathrm{C} \alpha$ distances of the $i$ th native contact in atomistic and CG copies, respectively. The force constant, $k_{i}$, can be different for various subsets of native contacts, for example, to emphasize the relative importance of tertiary contacts vs. local secondary ones. For large proteins with hundreds of native contacts, the simple harmonic potential of Eqn. 3 can lead to large penalty energies and result in severe exchange bottlenecks, particularly 
between the uncoupled $\left(\lambda_{1}=0\right)$ and coupled $(\lambda>0)$ conditions. For this, a restraint potential with a soft asymptote is used at large $\Delta d_{i}$,

$$
E_{i}\left(\Delta d_{i}\right)=A+\frac{B}{\left(\Delta d_{i}\right)^{s}}+f_{\max } \Delta d_{i}, \text { if } \Delta d_{i}>d_{s}
$$

In Eqn. $4, d_{s}$ is the distance threshold where the penalty function $E_{i}\left(\Delta d_{i}\right)$ switches from the harmonic form (Eqn. 3) to the soft asymptote. The switching exponent $s$ controls how quickly the limiting slope, $f_{\max }$, is approached at large $\Delta d_{i}$. The parameters $A$ and $B$ are identified by requiring both $E_{i}\left(\Delta d_{i}\right)$ and its first derivative to be continuous at the switching distance $\left(\Delta d_{i}=d_{s}\right)$. We note that similar penalty functions with soft asymptotes are widely employed in NMR structure calculations $^{28}$. The purpose is to avoid premature structural collapse due to a few large distance restraint violations and thus allow better conformational sampling to generate structures that are maximally consistent with all structural restraints. The simulations in the current work only involve exchanging a ladder of models with different $\lambda_{\mathrm{i}}$. More sophisticated Hamiltonian RE schemes can be devised that involve additional parameters of the restraint potentials, particularly $k_{\mathrm{i}}, d_{s}$ and $f_{\max }$, to more carefully control how conformational transitions are communicated between $\mathrm{CG}$ and atomistic copies for larger proteins.

The MSES method has been implemented in CHARMM ${ }^{29}$ and MMTSB ${ }^{30}$. Its efficacy is examined here using a series of $\beta$-hairpins derived from the protein G B1 domain, including GB1p (GEWTYD DATK TFTVTE), GB1m1 (GEWTYD DATK TATVTE), and, GB1m3 (KKWTYN PATG KFTVQE) (loop regions underlined and key mutations highlighted in bold fonts). The wildtype GB1p is $\sim 42 \%$ folded at $278 \mathrm{~K}$ based on NMR chemical shift analysis ${ }^{31}$. The Phe to Ala mutation in GB1m1 reduces the hairpin stability to $\sim 6 \%$ folded, and the more rigid prolinecontaining loop increases the stability of GB1m 3 to $\sim 86 \%$ folded $^{31}$. These $\beta$-hairpins, albeit small, resemble larger proteins in many essential aspects including cooperative folding transitions and microsecond folding timescales. The optimized generalized born with smooth switching (GBSW) atomistic implicit solvent force field appears to recapitulate both the structures and stabilities of these $\beta$-hairpins ${ }^{32}$. However, the previous T-RE simulations failed to generate converged conformational ensembles for GB $1 \mathrm{p}^{32}$. In fact, no reversible folding/unfolding transition was sampled by T-RE for any of these $\beta$-hairpins, and the apparent convergence for GB1m 1 and GB1m3 ensembles was mainly due to mixing of conformational states sampled at different temperatures. 
A sequence-flavored Gō-like model was first generated by the MMTSB Gō-Model Builder ${ }^{27}$ and then used in simulations of all three hairpins. The model represents each residue using a single $\mathrm{C} \alpha$ bead and adopts the Miyazawa-Jernigan (MJ) statistical potential ${ }^{33}$ for residue-specific native interactions. The Gō-like model was coupled to the GBSW atomistic implicit solvent model (Fig. 1a), by imposing the restraint potentials on all nine native contacts (see Eqns. 3-4). All MSES simulations were performed using 8 replicas with $\left\{\lambda_{i}, T_{i}\right\}=\{0,270\},\{0.05,290\},\{0.1,312\}$, $\{0.25,336\},\{0.4,361\},\{0.6,388\},\{0.8,418\}$, and $\{1.0,450\}$. These conditions were assigned by having roughly exponential distributions for both $\lambda$ and $T$. The exchange acceptance rates were uniform and $\sim 25 \%$ for all MSES simulations. Other parameters of the MSES restraint potential were: $k=1.0 \mathrm{kcal} / \mathrm{mol} / \AA^{2}, s=1, d_{s}=2.0 \AA$ and $f_{\max }=0.1 \mathrm{kcal} / \mathrm{mol} / \AA$. We note that the soft asymptote has minimal impacts on the exchange and sampling efficiencies for these small hairpins. Langevin simulations were performed with a friction coefficient of $0.1 \mathrm{ps}^{-1}$, and exchanges were attempted every 2 ps. For each peptide, two independent simulations were performed, starting from the folded (control) and fully extended (folding) structures, respectively. The length of all MSES simulations was $100 \mathrm{~ns}$ per replica. Control and folding T-RE simulations were also performed for GB1p as a reference. These simulations are summarized in Table 1.

We first validate if MSES can indeed recover the correct canonical ensembles at the uncoupled condition. Reliable reference atomistic ensembles are not available for these hairpins due to apparent difficulty in achieving convergence using T-RE simulations ${ }^{32}$. Therefore, we compare the CG ensembles derived from MSES simulations to a reference ensemble obtained from a 4- $\mu$ s T-RE simulation of the Gō-like model alone. Over 1300 reversible folding transitions were sampled in the T-RE simulation and the resulting reference $\mathrm{CG}$ ensemble at $270 \mathrm{~K}$ is fully converged. As illustrated in Fig 1b, MSES simulations generated CG ensembles virtually identical to the reference. That is, bias due to coupling between atomistic and CG models is completely removed via Hamiltonian RE as expected. The co-evolution of the CG and atomistic copies during MSES simulations is illustrated in Fig. S1. As designed, the CG and atomistic conformations can diverge at low temperature/weak coupling conditions, but strongly track one and another at high temperature/strong coupling conditions.

The efficacy of MSES in accelerating large-scale atomistic conformational transitions is first evaluated by calculating the number of reversible transitions between the folded ( $\geq 5$ native hydrogen bonds) and unfolded (no hydrogen bond) states at the atomistic level. Summarized in 
Table 1, the results show that T-RE simulations rarely sampled reversible folding transitions, with only one such event observed in the control and folding runs of GB1p. In contrast, many reversible transitions ( 31 on average) were sampled in MSES simulations of all $\beta$-hairpins, reflecting almost two orders of magnitude enhancement in the efficiency of sampling atomistic transitions. Fig. 2 depicts the evolution of the number of native hydrogen bonds from representative T-RE and MSES replicas. The T-RE replicas remain at either the folded or unfolded states throughout the simulation (Fig. 2a); whereas all MSES replicas undergo rapid reversible transitions between the folded and unfolded states (Fig. 2b), apparently driven by coupling to the efficient Gō-like model. To further evaluate the effects of MSES coupling on folding kinetics and efficiency, we performed multiple sets of folding simulations of GB1p at $270 \mathrm{~K}$ using the GBSW, Gō-like, and hybrid potentials (Eqn. 1). 100 50-ns simulations were initiated from unfolded atomistic and CG structures randomly selected from pre-generated equilibrium ensembles (see Table S1 and Fig. S2). The results show that coupling with the CG model allowed the atomistic model to reach the folded state in $34 \%$ of the trajectories within <18 ns on average, while all simulations in GBSW alone were trapped in various compact states and failed to fold within $50 \mathrm{~ns}$. The dramatic improvement in atomistic folding efficiency was achieved with only a moderate $50 \%$ increase in the average folding time of the $\mathrm{CG}$ model (from 0.83 to $1.19 \mathrm{~ns}$ ). Curiously, we observed that the CG model actually also underwent faster reversible folding transitions in MSES simulations, with an average rate of $k_{\mathrm{TS}} \sim 0.084 \mathrm{~ns}^{-1}$ compared to $k_{\mathrm{TS}} \sim 0.041 \mathrm{~ns}^{-1}$ in the T-RE simulation of the Gō-like model alone. This is likely because that coupling with the atomistic model accelerates unfolding transitions at the CG level.

Ideally, a fully converged RE simulation should involve all replicas sampling the same entire accessible conformational space. This limit is almost never achieved in atomistic T-RE simulations of nontrivial peptides and proteins, where individual replicas generally sample separate major conformational states throughout the simulations (e.g., see Fig. 3a-c). Amazingly, MSES simulations of all three $\beta$-hairpins display many characteristics of full convergence, with all replicas sampling similar, complete atomistic conformational spaces (Fig.3e-g and Fig. S3). When combining information from all replicas, T-RE and MSES simulations appear to cover similar conformational spaces (Fig. 3d and 3h), even though T-RE under samples most regions except the major free energy basins. Atomistic conformational ensembles derived from independent control and folding runs were compared to further evaluate the ability of MSES to achieve convergence in key thermodynamic properties. Fig. 4 compares the probability distributions of the number of native 
hydrogen bonds. The results show that MSES does generate largely consistent ensembles from the control and folding runs. Especially for GB1p, large discrepancies that persist between control and folding T-RE runs (Fig. 4a) are greatly reduced (Fig. 4b). We also note that, although the same Gōlike model was used for all three $\beta$-hairpins, the MSES simulations were able to recapitulate varied stabilities of these sequences in the GBSW implicit solvent as expected. Nonetheless, despite impressive conformational space coverage by all replicas in MSES simulations (Fig.3e-g and Fig. S3), substantial differences persist in ensembles derived from control and folding MSES runs, except for GB1m3 with a more rigid proline-containing loop. This illustrates the formidable challenges in generating converged equilibrium ensembles even for small but flexible peptides.

In conclusion, we have developed an effective MSES approach that utilizes efficient topology-based CG models to accelerate the sampling of complex and rough atomistic energy landscapes. The CG and atomistic model are coupled using native contact-based restraint potentials that are motivated by the current understanding of protein folding mechanisms as well as lessons from NMR structure calculations and allow excellent scalability to larger and more complex systems. The flexible and soft nature of the coupling potentials (Eqns. 3 and 4) also provides robust tolerance of moderate discrepancies in folding pathways at CG and atomistic levels, including potential involvement of non-native interactions. The bias from the coupling potential is removed by performing Hamiltonian/temperature RE, allowing one to benefit simultaneously from faster transitions of the CG model and the accuracy of the atomistic force field. Application to implicit solvent simulations of small but nontrivial GB1p series of $\beta$-hairpins demonstrates that MSES dramatically accelerate atomistic folding/unfolding transitions and improves the convergence of various thermodynamic properties of interest. We anticipate MSES to be highly useful whenever generation of wellconverged protein conformational ensembles is critical, including intensive current efforts that rely on peptide simulations to optimize implicit and explicit solvent protein force fields ${ }^{34,35}$.

\section{Acknowledgement}

This work was supported by the National Science Foundation (MCB 0952514). Part of the computing for this project was performed on the Beocat Research Cluster at Kansas State University, which is funded in part by NSF grants CNS-1006860, EPS-1006860, and EPS-0919443. This work is contribution number 14-176-J from the Kansas Agricultural Experiment Station. 
Supporting Information Available: Three figures and a table showing coevolution of CG and atomistic models, conformational coverage, and folding kinetics and efficiency of CG and atomistic models in MSES. This material is available free of charge via the Internet at http://pubs.acs.org. 


\section{References}

1. Lei, H.; Duan, Y., Improved Sampling Methods for Molecular Simulation. Curr. Opin. Struct. Biol. 2007, 17, 187-91.

2. Swendsen, R. H.; Wang, J. S., Replica Monte-Carlo Simulation of Spin-Glasses. Phys. Rev. Lett. 1986, 57, 2607-2609.

3. Sugita, Y.; Okamoto, Y., Replica-Exchange Molecular Dynamics Method for Protein Folding. Chem. Phys. Lett. 1999, 314, 141-151.

4. Hansmann, U. H. E.; Okamoto, Y., Numerical Comparisons of Three Recently Proposed Algorithms in the Protein Folding Problem. J. Comput. Chem. 1997, 18, 920-933.

5. Zheng, W.; Andrec, M.; Gallicchio, E.; Levy, R. M., Simulating Replica Exchange Simulations of Protein Folding with a Kinetic Network Model. Proc. Natl. Acad. Sci. U. S. A. 2007, 104, 15340-5.

6. Zuckerman, D. M.; Lyman, E., A Second Look at Canonical Sampling of Biomolecules Using Replica Exchange Simulation. J. Chem. Theory Comput. 2006, 2, 1200-1202.

7. Periole, X.; Mark, A. E., Convergence and Sampling Efficiency in Replica Exchange Simulations of Peptide Folding in Explicit Solvent. J. Chem. Phys. 2007, 126, 014903.

8. Sindhikara, D.; Meng, Y. L.; Roitberg, A. E., Exchange Frequency in Replica Exchange Molecular Dynamics. J. Chem. Phys. 2008, 128.

9. Nymeyer, H., How Efficient Is Replica Exchange Molecular Dynamics? An Analytic Approach. J. Chem. Theory Comput. 2008, 4, 626-636.

10. Kim, J.; Keyes, T.; Straub, J. E., Generalized Replica Exchange Method. J. Chem. Phys. 2010, 132, 224107.

11. Denschlag, R.; Lingenheil, M.; Tavan, P., Efficiency Reduction and Pseudo-Convergence in Replica Exchange Sampling of Peptide Folding-Unfolding Equilibria. Chem. Phys. Lett. 2008, 458, 244-248.

12. Zhang, W.; Chen, J., Efficiency of Adaptive Temperature-Based Replica Exchange for Sampling Large-Scale Protein Conformational Transitions. J. Chem. Theory Comput. 2013, 9 , 2849-2856.

13. Kubelka, J.; Hofrichter, J.; Eaton, W. A., The Protein Folding 'Speed Limit'. Curr. Opin. Struct. Biol. 2004, 14, 76-88.

14. Bicout, D. J.; Szabo, A., Entropic Barriers, Transition States, Funnels, and Exponential Protein Folding Kinetics: A Simple Model. Protein Sci. 2000, 9, 452-65.

15. Schuler, B.; Lipman, E. A.; Eaton, W. A., Probing the Free-Energy Surface for Protein Folding with Single-Molecule Fluorescence Spectroscopy. Nature 2002, 419, 743-747. 
16. Beck, D. A.; White, G. W.; Daggett, V., Exploring the Energy Landscape of Protein Folding Using Replica-Exchange and Conventional Molecular Dynamics Simulations. J. Struct. Biol. 2007, 157, 514-23.

17. Ayton, G. S.; Noid, W. G.; Voth, G. A., Multiscale Modeling of Biomolecular Systems: In Serial and in Parallel. Curr. Opin. Struct. Biol. 2007, 17, 192-198.

18. Lwin, T. Z.; Luo, R., Overcoming Entropic Barrier with Coupled Sampling at Dual Resolutions. J. Chem. Phys. 2005, 123, 194904.

19. Lyman, E.; Ytreberg, F. M.; Zuckerman, D. M., Resolution Exchange Simulation. Phys. Rev. Lett. 2006, 96, 028105.

20. Moritsugu, K.; Terada, T.; Kidera, A., Scalable Free Energy Calculation of Proteins Via Multiscale Essential Sampling. J. Chem. Phys. 2010, 133, 224105.

21. Liu, P.; Kim, B.; Friesner, R. A.; Berne, B. J., Replica Exchange with Solute Tempering: A Method for Sampling Biological Systems in Explicit Water. Proc. Natl. Acad. Sci. U. S. A. 2005, $102,13749-13754$.

22. Kwak, W.; Hansmann, U. H., Efficient Sampling of Protein Structures by Model Hopping. Phys. Rev. Lett. 2005, 95, 138102.

23. Meinke, J. H.; Hansmann, U. H., Protein Simulations Combining an All-Atom Force Field with a Go Term. J. Phys.: Condens. Matter 2007, 19, 285215.

24. Liu, P.; Voth, G. A., Smart Resolution Replica Exchange: An Efficient Algorithm for Exploring Complex Energy Landscapes. J. Chem. Phys. 2007, 126, 045106.

25. Hills, R. D.; Brooks, C. L., Insights from Coarse-Grained Go Models for Protein Folding and Dynamics. Int. J. Mol. Sci. 2009, 10, 889-905.

26. Wolynes, P. G., Recent Successes of the Energy Landscape Theory of Protein Folding and Function. Q. Rev. Biophys. 2005, 38, 405-410.

27. Karanicolas, J.; Brooks, C. L., The Origins of Asymmetry in the Folding Transition States of Protein L and Protein G. Protein Sci. 2002, 11, 2351-2361.

28. Brunger, A. T.; Nilges, M., Computational Challenges for Macromolecular Structure Determination by X-Ray Crystallography and Solution Nmr-Spectroscopy. Q. Rev. Biophys. 1993, 26, 49-125.

29. Brooks, B. R.; Brooks, C. L.; Mackerell, A. D.; Nilsson, L.; Petrella, R. J.; Roux, B.; Won, Y.; Archontis, G.; Bartels, C.; Boresch, S.; Caflisch, A.; Caves, L.; Cui, Q.; Dinner, A. R.; Feig, M.; Fischer, S.; Gao, J.; Hodoscek, M.; Im, W.; Kuczera, K.; Lazaridis, T.; Ma, J.; Ovchinnikov, V.; Paci, E.; Pastor, R. W.; Post, C. B.; Pu, J. Z.; Schaefer, M.; Tidor, B.; Venable, R. M.; Woodcock, H. L.; Wu, X.; Yang, W.; York, D. M.; Karplus, M., Charmm: The Biomolecular Simulation Program. J. Comput. Chem. 2009, 30, 1545-1614. 
30. Feig, M.; Karanicolas, J.; Brooks, C. L., Mmtsb Tool Set: Enhanced Sampling and Multiscale Modeling Methods for Applications in Structural Biology. J. Mol. Graphics Modell. 2004, 22, 377-395.

31. Fesinmeyer, R. M.; Hudson, F. M.; Andersen, N. H., Enhanced Hairpin Stability through Loop Design: The Case of the Protein G B1 Domain Hairpin. J. Am. Chem. Soc. 2004, 126, 723843.

32. Chen, J. H.; Im, W. P.; Brooks, C. L., Balancing Solvation and Intramolecular Interactions: Toward a Consistent Generalized Born Force Field. J. Am. Chem. Soc. 2006, 128, 3728-3736.

33. Miyazawa, S.; Jernigan, R. L., Residue-Residue Potentials with a Favorable Contact Pair Term and an Unfavorable High Packing Density Term, for Simulation and Threading. J. Mol. Biol. 1996, 256, 623-644.

34. Chen, J. H., Effective Approximation of Molecular Volume Using Atom-Centered Dielectric Functions in Generalized Born Models. J. Chem. Theory Comput. 2010, 6, 2790-2803.

35. Best, R. B.; Zhu, X.; Shim, J.; Lopes, P. E. M.; Mittal, J.; Feig, M.; MacKerell, A. D., Optimization of the Additive Charmm All-Atom Protein Force Field Targeting Improved Sampling of the Backbone $\Phi, \Psi$ and Side-Chain X1 and X2 Dihedral Angles. J. Chem. Theory Comput. 2012, $8,3257-3273$. 


\section{Tables}

Table 1. Summary of T-RE and MSES simulations of GB1p, GB1m1 and GB1m3. All simulations involve 8 replicas and last $100 \mathrm{~ns}$. $N_{\mathrm{TS}}$ is the number of reversible folding transitions sampled by all replicas during the entire course of the simulation. The effective reversible transition rate $k_{\mathrm{TS}}$ is calculated as $N_{\text {TS }}$ divided by total simulation time ( $800 \mathrm{~ns}$ in all cases).

\begin{tabular}{ccccc}
\hline Sequence & Protocol & Run & $\boldsymbol{N}_{\text {TS }}$ & $\mathbf{k}_{\text {TS }}\left(\mathbf{n s} \mathbf{s}^{-\mathbf{1}}\right)$ \\
\hline GB1p & T-RE & Control & 1 & 0.0013 \\
& & Folding & 0 & - \\
GB1p & MSES & Control & 43 & 0.054 \\
& & Folding & 33 & 0.041 \\
GB1m1 & MSES & Control & 32 & 0.040 \\
& & Folding & 20 & 0.025 \\
GB1m3 & MSES & Control & 34 & 0.043 \\
& & Folding & 26 & 0.033 \\
\hline
\end{tabular}




\section{Figures and Figure Captions}

a

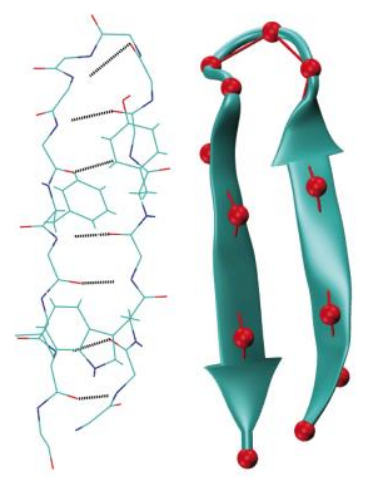

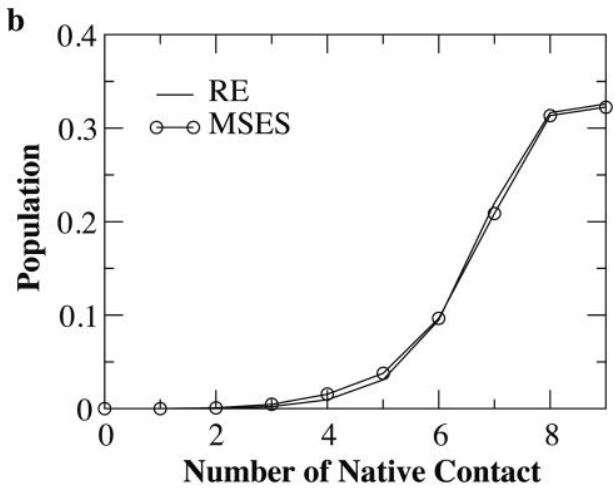

Fig. 1. Folded structure and simulated ensembles of GB1p. a) Left: Atomistic structure of GB1p. Dashed lines indicate all seven native hydrogen bonds. Right: Hybrid model containing atomistic (cyan cartoon) and $\mathrm{C} \alpha$-only CG (red beads) copies. b) Probability distributions of the number of native contacts for $\mathrm{CG}$ ensembles derived from a reference $\mathrm{T}-\mathrm{RE}$ simulation of the Gō model alone (solid line) and the MSES control simulation of GB1p (solid line with circles).

a. T-RE

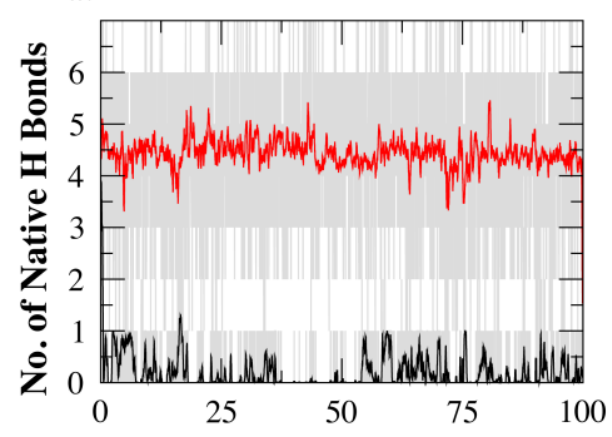

b. MSES

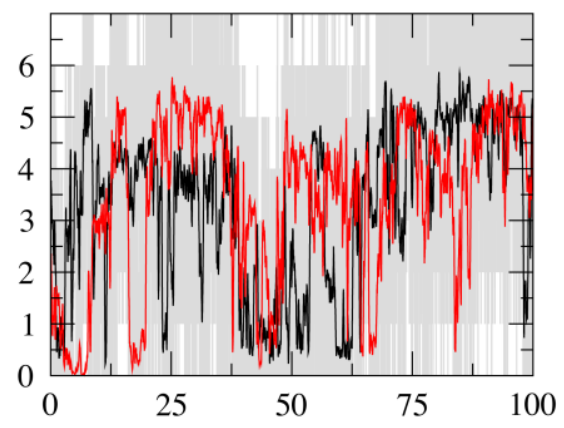

Time (ns)

Fig. 2. Numbers of native hydrogen bonds as a function of time for representative replicas from a) T-RE and b) MSES control simulations of GB1p. Gray lines depict the raw data, and black and red lines show the 50-ps running averages. 

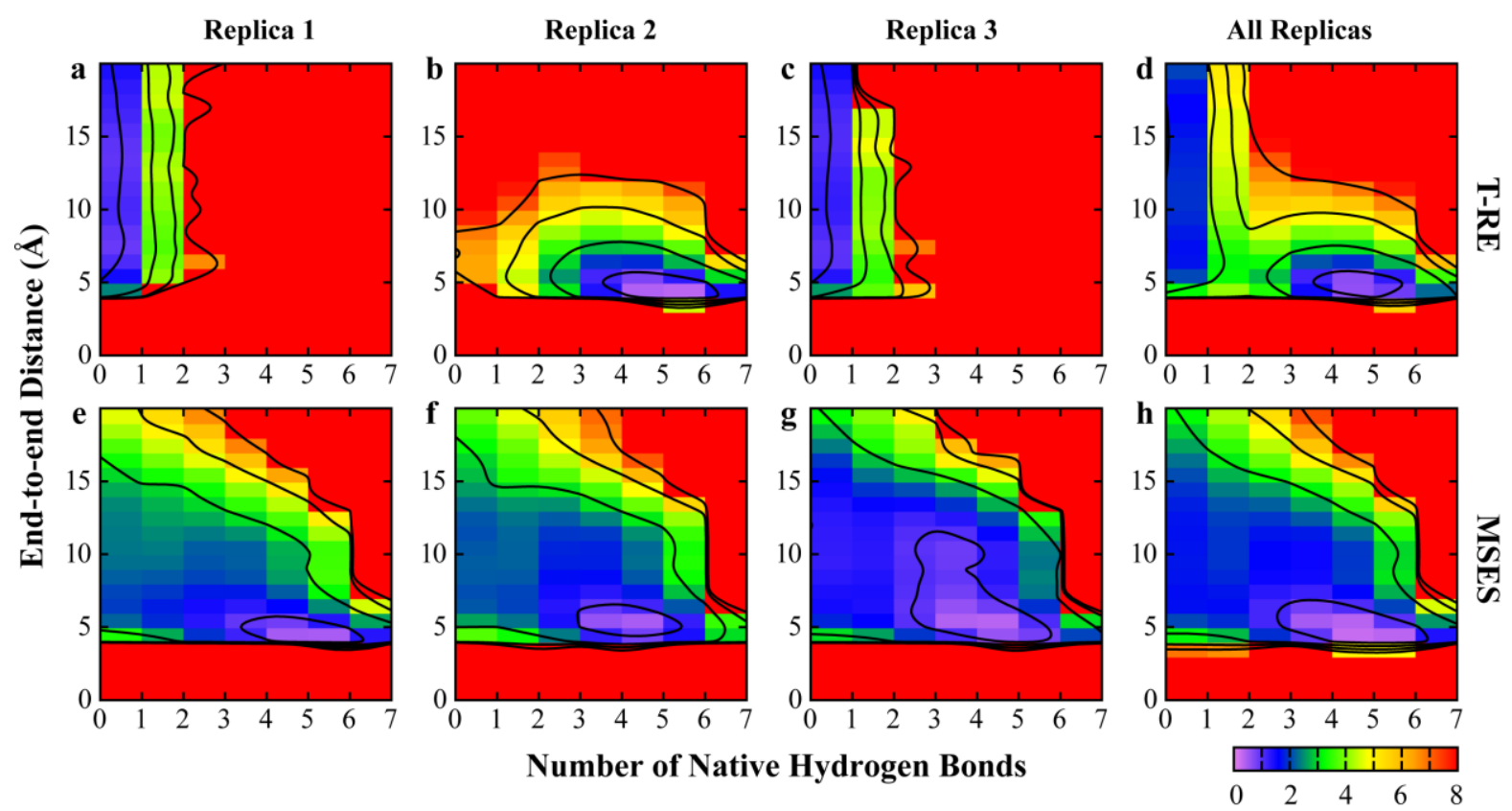

Fig. 3. Conformational space sampled by individual replicas in the T-RE (a-c) and MSES (e-g) control simulations of GB1p. Panels d) and h) were calculated by including all replicas. All atomistic conformations sampled during the last $80 \mathrm{~ns}$ of these simulations, regardless of the temperature or coupling scaling factor, were included to compute the pseudo-free energy surfaces, which are plot in the unit of kT. Contours are shown at 1, 3, 5, and 7 kT levels.

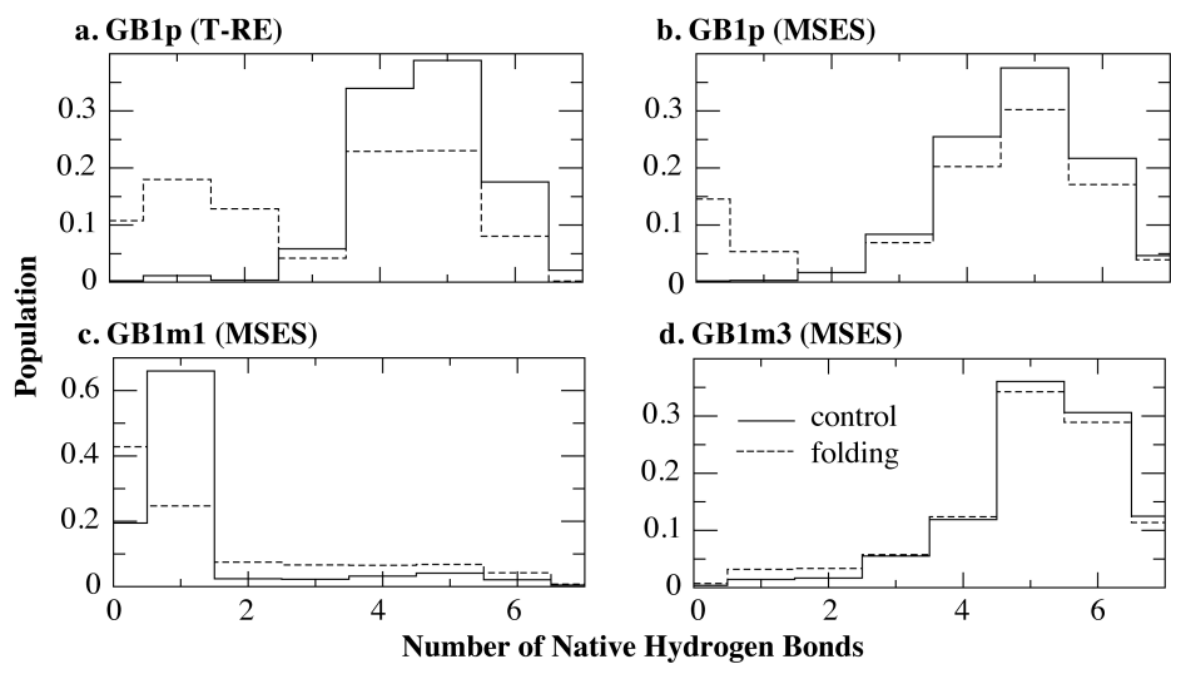

Fig.4. Probability distributions of the number of native hydrogen bonds for GB1p (a-b), GB1m1 (c), and GB1m3 (d). These distributions were calculated from structure ensembles extracted from the last $80 \mathrm{~ns}$ of T-RE or MSES simulations at $T=270 \mathrm{~K}$ (and $\lambda=0$ ) (see Table 1 for detailed simulation setups). 
TOC Graphic
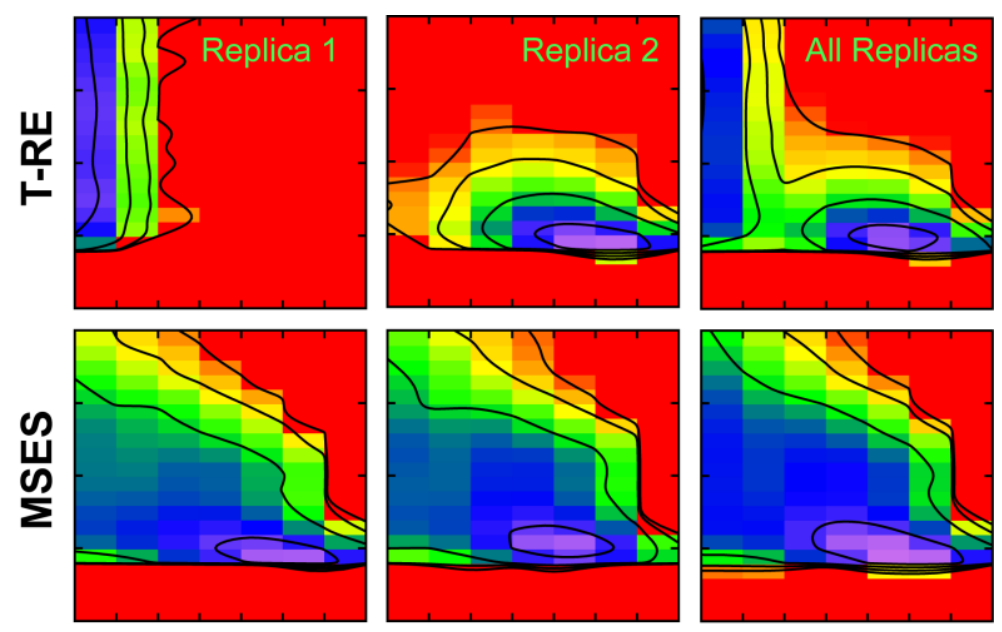